\title{
Synthesis of pyrazole-4-carbohydrazide derivatives of pharmaceutical interest
}

\author{
Giuseppe Daidone*, Demetrio Raffa, Fabiana Plescia, Benedetta Maggio, and Angela \\ Roccaro
}

Dipartimento di Chimica e Tecnologie Farmaceutiche

Via Archirafi, 3- I-90123 Palermo, Italy

E-mail: gdaidone@unipa.it

\section{This paper is dedicated to Professor Domenico Spinelli on the occasion of his $70^{\text {th }}$ birthday (received 23 Oct 02; accepted 26 Dec 02; published on the web 03 Jan 03)}

\begin{abstract}
New 1-phenyl- or 1-methyl-5-benzamidopyrazole-4-carbohydrazide derivatives were prepared in 70-90\% yields from 1-methyl- or 1-phenyl-6-phenylpyrazolo[3,4-d]1,3-oxazin-4(1H)-one derivatives and hydrazine hydrate. Small quantities of the isomeric 5-aminopyrazole-4- $(N-$ benzoyl)hydrazides were detected in some reaction mixtures, proving that intramolecular benzoyl migration can take place in the 5-benzamidopyrazole-4-carbohydrazide molecule. The direct formation of pyrazole-4-carbohydrazides from 5-benzamidopyrazole-4-carboxylic acid ethyl esters and hydrazine hydrate was unsuccessful.
\end{abstract}

Keywords: Pyrazole-4-carbohydrazides, pyrazole-4-( $N$-benzoyl)carbohydrazides, intramolecular benzoyl migration

\section{Introduction}

In the course of our medicinal chemistry researches, we needed to prepare pyrazole-4carbohydrazide derivatives $\mathbf{2 a - h}$ (Schemes 1 and 2) in view of their potential pharmacological activities. In the literature it is reported that some benzohydrazides are used to inhibit fibrosis and to treat fibrosing disorder. ${ }^{1}$ Several aryl- and heteroaryl- hydrazides produce inhibitory effects on glutamic acid decarboxylase (GAD), GABA- $\alpha$-oxoglutarate amino transferase (GABA-T) and monoamine oxidase. ${ }^{2,3}$ Moreover, Isoniazid ${ }^{\circledR}$, namely pyridine-4-carbohydrazide, is the drug of choice in the treatment of tubercolosis. ${ }^{4}$

A review of the literature ${ }^{5}$ revealed that 1-phenyl-5-benzamidopyrazole-4-carboxylic acid ethyl ester 1 (Scheme 1) when refluxed for $5 \mathrm{~h}$ in hydrazine hydrate afforded the 5-amino derivative $\mathbf{3}$, by losing the benzoyl moiety, instead of the hydrazide derivative 2a. 


\section{Results and Discussion}

On the basis of the literature data, we heated the above mixture under reflux for $1 \mathrm{~h}$, obtaining the same result. At this point the formation of 2a under other reaction conditions was attempted: an ethanolic solution of 1-phenyl-5-benzamidopyrazole-4-carboxylic acid ethyl ester and hydrazine hydrate in molar ratio 1:5 was heated under reflux for 15 hours, but TLC showed that the ester was not transformed. The experiment was repeated under acid catalysis, but the desired product 2a was not obtained.<smiles>CCOC(=O)c1cnn(-c2ccccc2)c1NC(=O)c1ccccc1</smiles>

1<smiles>C[Y](=O)c1cnn(-c2ccccc2)c1NC(=O)c1ccccc1</smiles>

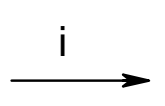

i; pure hydrazine hydrate, reflux ii; hydrazine hydrate in ethanol, $\mathrm{H}^{+}$, reflux

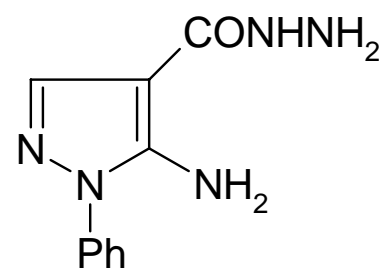

3

\section{Scheme 1}

Previously we have reported $^{6}$ that the reaction of pyrazole[3,4- $\left.d\right]$-1,3-oxazin-4-one derivatives of type $\mathbf{4}$ with anilines afforded, in high yields, a number of phenylamides of 5benzamidopyrazole-4-carboxylic acids. In analogy with this reaction we reacted the pyrazole[3,4- $d$ ]-1,3-oxazinones $\mathbf{4 a - h}$ with hydrazine hydrate, hoping to obtain the pyrazole-4carbohydrazides $\mathbf{2 a - h}$. When the pyrazolo-oxazinone 4a was reacted with hydrazine hydrate, a product was obtained in $75 \%$ yield, together with a small amount of a second one. On the basis of elemental analysis and molecular weight (MS), they proved to be isomers. We assigned structure 2a to the high-yield product and structure 5a to the isomer (Scheme 2). 


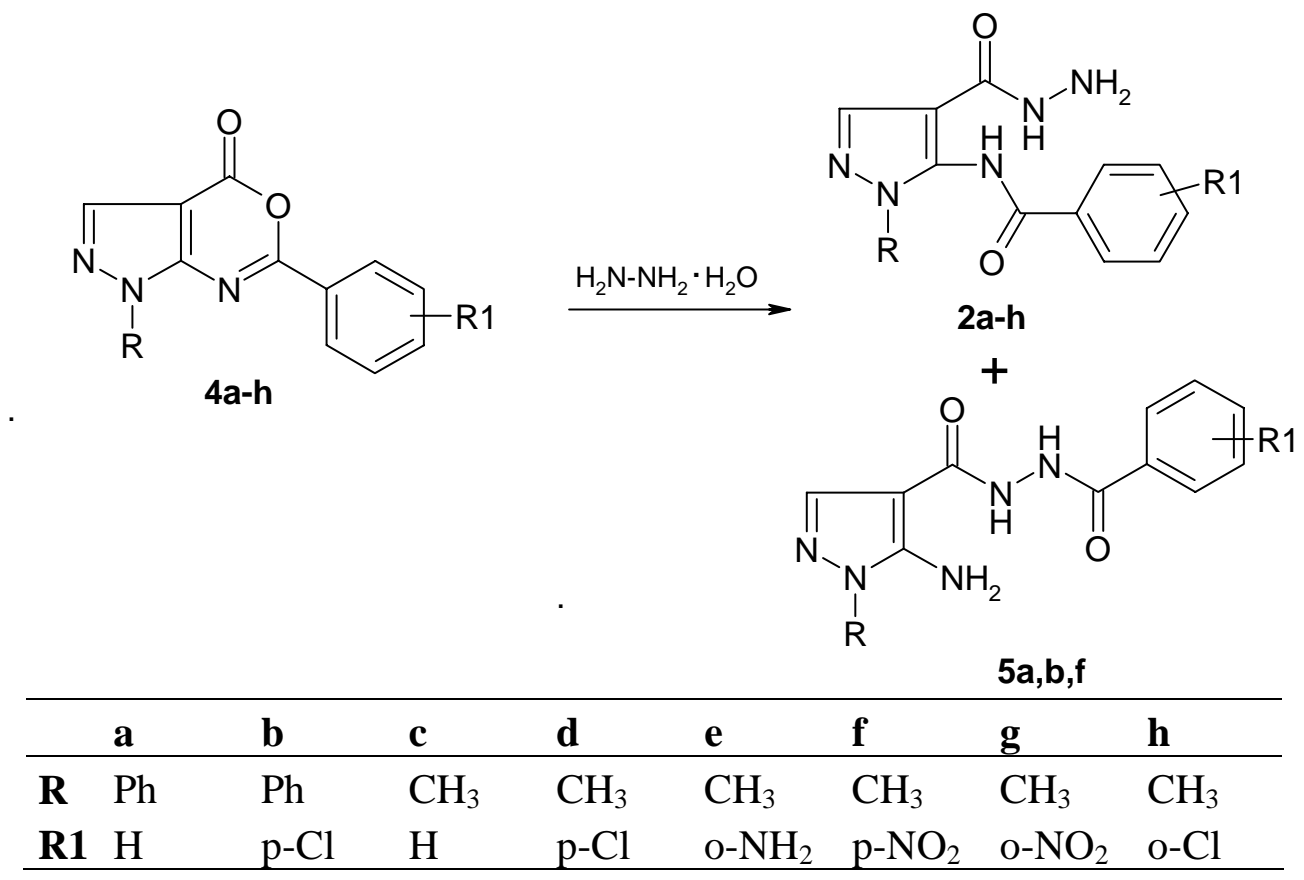

\section{Scheme 2}

The attribution of the structures was based on NMR data. The ${ }^{1} \mathrm{H}-\mathrm{NMR}$ spectrum of the major product showed a signal for two hydrogens at $\delta 4.39$, exchangeable with $\mathrm{D}_{2} \mathrm{O}$, and the minor isomer produced the same signal at $\delta 6.40$; the low-field signal is attributable to the amino group bonded to the pyrazole nucleus, and the signal at $\delta 4.39$ to the more shielded hydrazide amino group. The latter value is in accordance with the signal reported in the literature for the hydrazide amino group of 2-pyridinecarbohydrazide. ${ }^{7}$ The two NH signals of 5a (9.96 and 10.36 $\delta$ ) were closer than those of $\mathbf{2 a}(9.43$ and $10.40 \delta$ ) owing to a more similar environment of the $\mathrm{NH}$ groups in 5a. In order to confirm the structure 5a, the 1-phenyl-5-aminopyrazole-4carbohydrazide 3a and benzoyl chloride 6a were reacted (Scheme 3), affording a compound identical in all respects to 5 a.

Moreover, reaction of this product 5a with ethyl orthoformate gave the 1-phenyl-5benzamido-1H-pyrazolo[3,4- $d$ ]pyrimidine-4-one 7a which was also obtained by benzoylation of the 5-amino-1H-pyrazolo[3,4- $d$ ]pyrimidine-4-one $\mathbf{8}^{8}$ At this point, the other pyrazolo[3,4$d$ ] oxazinones $\mathbf{4 b}$-h were reacted with hydrazine hydrate affording the related pyrazolo-4carbohydrazides $\mathbf{2 b - h}$ in $70-90 \%$ yields. The NMR spectra of these compounds showed the signal for the hydrazide amino group in the $\delta 4.32-4.39$ range and those for the two NH groups at $\delta$ 9.20-10.79. The reaction mixtures of $\mathbf{2} \mathbf{b}-\mathbf{h}$ were monitored for the presence of $\mathbf{5} \mathbf{b}-\mathbf{h}$ by ${ }^{1} \mathbf{H}-$ NMR. On the basis of the 5-amino signal, the spectra revealed the presence of small amounts of 5b,f (signals at 6.44 and $6.25 \delta$ respectively). These results were confirmed by TLC using authentic specimens of $\mathbf{5 b}$,f as reference compounds. Compounds $\mathbf{5 b}$,f were synthesized as for 5a (Scheme 3) and identified by elemental and spectroscopic data. The ${ }^{1} \mathrm{H}-\mathrm{NMR}$ spectra of $\mathbf{5 b , f}$ 
showed the 5-amino signal at 6.45 and $6.22 \delta$, respectively. The formation of $\mathbf{2 a - h}$ can easily be explained by a nucleophilic attack on the carbonyl group of the oxazinone ring of $\mathbf{4 a - h}$. It appears that this process can be followed by intramolecular migration of a benzoyl group in compounds 2a,b,f to give the isomers 5a,b,f, whose formation is rationalized in Scheme 4.<smiles>[R]n1ncc(C(N)=O)c1N</smiles>

$3 a, f$<smiles></smiles>

8<smiles>[R1]c1ccccc1C(=O)Cl</smiles>

$6 a, b, f$<smiles>[CH]C</smiles><smiles>[R1]c1cccc(C(=O)NNC(=O)c2cnn([R])c2N)c1</smiles><smiles>O=C(Cl)c1ccccc1</smiles>

$6 a$<smiles>Cc1nc2c(cnn2-c2ccccc2)c(=O)n1NC(=O)c1ccccc1</smiles>

$7 a$

\section{Scheme 3}
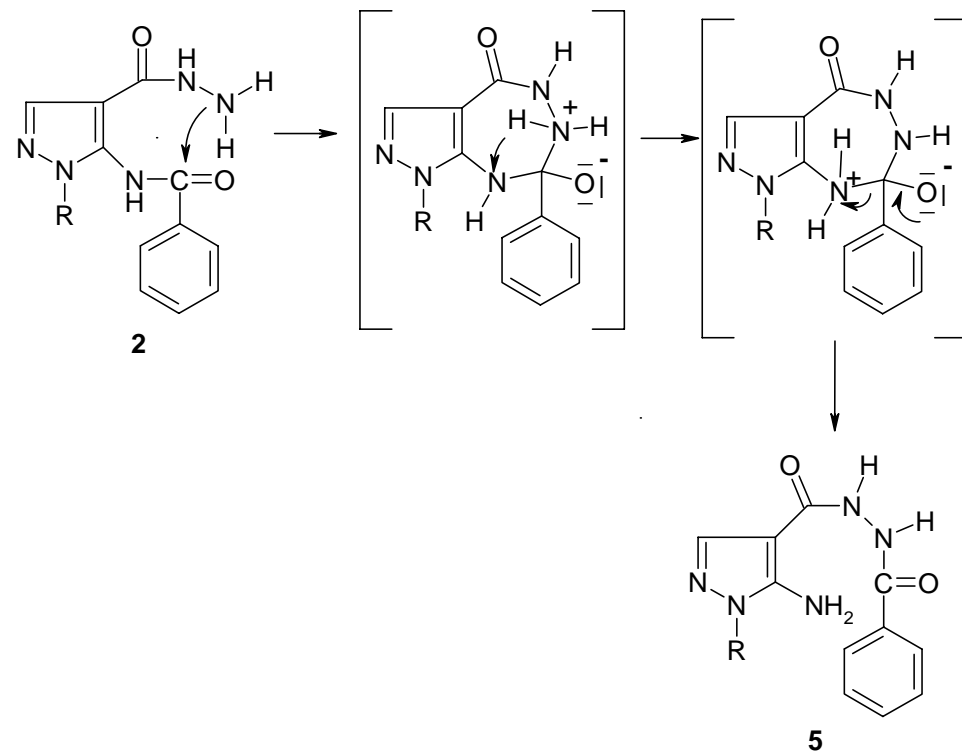

Scheme 4 
Lastly, the hitherto unknown pyrazolo-oxazinones $\mathbf{4 b , d , f}$ were synthesized following Scheme 5. The structures of the above compounds, as well as those of the intermediate derivatives $\mathbf{1 0}$ and $\mathbf{1 1}$ were assigned on the basis of satisfactory elemental and spectroscopic data.

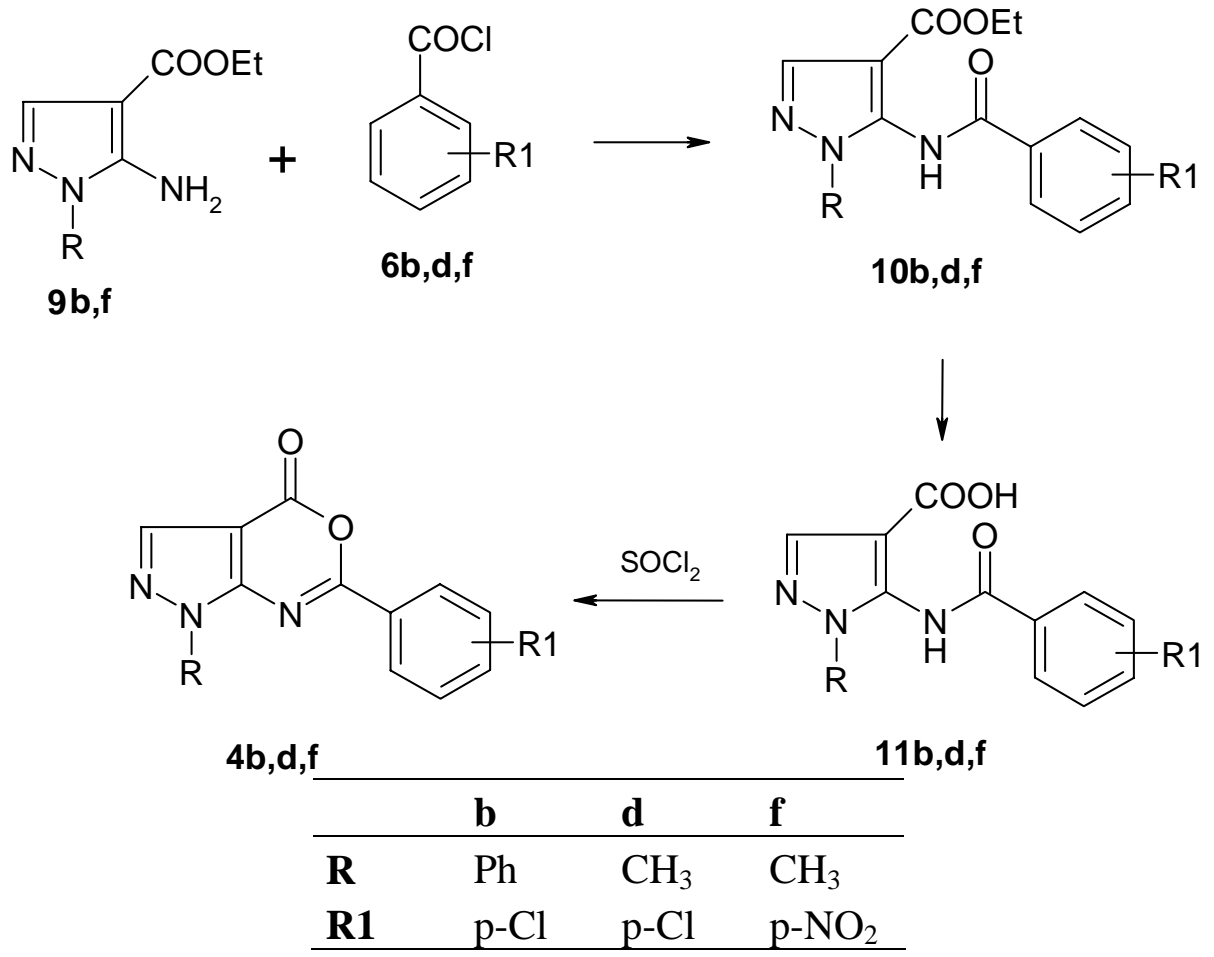

\section{Scheme 5}

\section{Experimental Section}

General Procedures. Melting points were determined on a Büchi 530 capillary melting point apparatus and are uncorrected; IR spectra were recorded in Nujol mulls with a Jasco IR-810 spectrophotometer; ${ }^{1} \mathrm{H}-\mathrm{NMR}$ spectra were obtained in $\mathrm{DMSO}^{-\mathrm{d}_{6}}$ on a Bruker AC-E $250 \mathrm{MHz}$ spectrometer (TMS as internal standard). Mass measurements at low resolution were obtained on an Autospect Ultima Orthogonal T.O.F.T. mass spectrometer operating at $75 \mathrm{eV}$. Elemental analyses $(\mathrm{C}, \mathrm{H}, \mathrm{N})$, performed by Dipartimento di Scienze Farmaceutiche-Università di Catania, were within $\pm 0.4 \%$ of theoretical values.

Reaction of 1-(R)-6-(substituted)phenyl-1H-pyrazolo[3,4-d]1,3-oxazin-4-one derivatives 4a$h$ with hydrazine hydrate. General procedure for 1- $R$-5-(substituted)benzamidopyrazole-4carbohydrazides $\mathbf{2 a - h . ~} \mathbf{4 a , c , e , g , h}{ }^{9}$ and $\mathbf{4 b , d , f}(6 \mathrm{mmol})$ were reacted under reflux for $2 \mathrm{~h}$ with 6 mmol of hydrazine hydrate $(0.3 \mathrm{ml}$ of $99 \%$ solution) in absolute ethanol (40 ml). The mixture, containing $\mathbf{2} \mathbf{c}-\mathbf{h}$, was then allowed to cool at room temperature. The precipitate was filtered off, 
and recrystallized from ethanol to give $\mathbf{2} \mathbf{c}-\mathbf{h}$. In the case of $\mathbf{2 b}$ the mixture was evaporated under vacuum and the solid recrystallized from ethyl acetate, whereas for 2a the precipitate was obtained after scratching. Yields $70-90 \%$. The mother liquors of 2a were evaporated and the residue was chromatographed by flash chromatography: ${ }^{10}$ silica gel (230-400 mesh), external column diameter $3.5 \mathrm{~cm}$, ethyl acetate as eluent, fractions each $50 \mathrm{ml}$. The fractions 6-11 were collected and evaporated under reduced pressure to give $70 \mathrm{mg}$ of pure 1-phenyl-5-amino- $1 \mathrm{H}$ pyrazole-4-(N-benzoyl)carbohydrazide 5a. 2a (75\%); m.p. 183-185 ${ }^{\circ} \mathrm{C}$; MS $(\mathrm{m} / \mathrm{z})$ : $321\left(\mathrm{M}^{+}\right)$; IR ( $\mathrm{cm}^{-1}$ ): 3440-3115 (multiple bands, $\mathrm{NH}_{2}$, and 2xNH), 1675-1625 (multiple bands, $2 \mathrm{xCO}$ ); ${ }^{1} \mathrm{H}-$

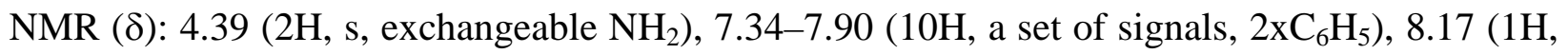
s, pyrazole H-3), $9.43(1 \mathrm{H}, \mathrm{s}$, exchangeable $\mathrm{NH}), 10.40$ (1H, s, exchangeable NH). 5a m.p. 255$258{ }^{\circ} \mathrm{C}$; MS $(\mathrm{m} / \mathrm{z}): 321\left(\mathrm{M}^{+}\right)$; IR $\left(\mathrm{cm}^{-1}\right)$ : 3450-3100 (multiple bands, $\mathrm{NH}_{2}$ and 2xNH), 1670, 1620 (br., 2xCO); ${ }^{1} \mathrm{H}-\mathrm{NMR}(\delta): 6.48\left(2 \mathrm{H}\right.$, s, exchangeable $\left.\mathrm{NH}_{2}\right), 7.39-7.98(10 \mathrm{H}$, a set of signals, $\left.2 \mathrm{xC}_{6} \mathrm{H}_{5}\right), 8.12(1 \mathrm{H}, \mathrm{s}$, pyrazole $\mathrm{H}-3), 10.02(1 \mathrm{H}, \mathrm{s}$, exchangeable $\mathrm{NH}), 10.42(1 \mathrm{H}, \mathrm{s}$, exchangeable NH). 2b (70\%); m.p. $182-183{ }^{\circ} \mathrm{C}$; MS (m/z): $355\left(\mathrm{M}^{+}\right)$; IR $\left(\mathrm{cm}^{-1}\right)$ : 3400-3100 (multiple bands, $\left.\mathrm{NH}_{2}, 2 \mathrm{xNH}\right), 1665,1638$ (2xCO); ${ }^{1} \mathrm{H}-\mathrm{NMR}(\delta)$ : 4.41 (2H, s, exchangeable $\mathrm{NH}_{2}$ ) 7.37-7.91 (9H, a set of signals, $\mathrm{C}_{6} \mathrm{H}_{5}$ and $\left.\mathrm{C}_{6} \mathrm{H}_{4}\right) 8.18(1 \mathrm{H}$, s, pyrazole $\mathrm{H}-3), 9.45(1 \mathrm{H}, \mathrm{s}$, exchangeable $\mathrm{NH}), 10.49$ (1H, s, exchangeable $\mathrm{NH})$. 2c (80\%); m.p. 205-206 ${ }^{\circ} \mathrm{C}$; MS (m/z): 259 $\left(\mathrm{M}^{+}\right)$; IR $\left(\mathrm{cm}^{-1}\right)$ : 3450-3100 (multiple bands, $\mathrm{NH}_{2}, 2 \mathrm{xNH}$ ), 1670-1630 (multiple bands, 2xCO); ${ }^{1} \mathrm{H}-\mathrm{NMR}(\delta)$ : 3.67 (3H, s, $\left.\mathrm{CH}_{3}\right), 4.32$ (2H, s, exchangeable $\mathrm{NH}_{2}$ ) 7.53-8.04 (6H, a set of signals, $\mathrm{C}_{6} \mathrm{H}_{5}$ and pyrazole $\left.\mathrm{H}-3\right)$, $9.25(1 \mathrm{H}, \mathrm{s}$, exchangeable $\mathrm{NH}), 10.34(1 \mathrm{H}, \mathrm{s}$, exchangeable $\mathrm{NH}) .2 \mathbf{d}$ (70\%); m.p. 249-250 ${ }^{\circ} \mathrm{C}$; MS (m/z): $293\left(\mathrm{M}^{+}\right)$; IR $\left(\mathrm{cm}^{-1}\right)$ : 3400-3100 (NH2, 2xNH) 1705, 1635 (2xCO); ${ }^{1} \mathrm{H}-\mathrm{NMR}(\delta): 3.66$ (3H, s, $\left.\mathrm{CH}_{3}\right), 4.30$ (2H, s, exchangeable $\left.\mathrm{NH}_{2}\right), 7.62-8.05$ (5H, a set of signals, $\mathrm{C}_{6} \mathrm{H}_{4}$ and pyrazole $\left.\mathrm{H}-3\right), 9.24(1 \mathrm{H}$, s, exchangeable $\mathrm{NH}), 10.39(1 \mathrm{H}$, s, exchangeable NH). 2e (78\%); m.p. $208-209{ }^{\circ} \mathrm{C}$; MS ( $\left.\mathrm{m} / \mathrm{z}\right): 274\left(\mathrm{M}^{+}\right)$; IR $\left(\mathrm{cm}^{-1}\right)$ : 3420, 3290 (br., $2 x N H$, 2xNH${ }_{2}$ ) 1690, 1680, 1630 (2xCO); ${ }^{1} \mathrm{H}-\mathrm{NMR}(\delta): 3.65$ (3H, s, $\left.\mathrm{CH}_{3}\right), 4.35$ (2H, s, exchangeable $\left.\mathrm{NH}_{2}\right)$ 6.25-8.00 (7H, a set of signals, $\mathrm{C}_{6} \mathrm{H}_{4}$, pyrazole $\mathrm{H}-3$ and exchangeable $\left.\mathrm{NH}_{2}\right), 9.22(1 \mathrm{H}, \mathrm{s}$ exchangeable $\mathrm{NH}), 10.00$ (1H, s, exchangeable $\mathrm{NH}) .2 f(90 \%)$; m.p. $238-240{ }^{\circ} \mathrm{C}$; $\mathrm{MS}(\mathrm{m} / \mathrm{z}): 304$ $\left(\mathrm{M}^{+}\right)$; IR $\left(\mathrm{cm}^{-1}\right)$ : 3400-3100 (multiple bands, $2 \mathrm{xNH}, \mathrm{NH}_{2}$ ), 1681, 1632 (2xCO); ${ }^{1} \mathrm{H}-\mathrm{NMR}(\delta)$ : $3.68\left(3 \mathrm{H}, \mathrm{s}, \mathrm{CH}_{3}\right), 4.36\left(2 \mathrm{H}\right.$, br. s, exchangeable $\left.\mathrm{NH}_{2}\right), 7.89(1 \mathrm{H}, \mathrm{s}$, pyrazole $\mathrm{H}-3), 8.22-8.43$ (4H, dd, $\left.\mathrm{C}_{6} \mathrm{H}_{4}\right), 9.29$ (s, $1 \mathrm{H}$, exchangeable $\left.\mathrm{NH}\right), 10.67$ (1H, br. s, exchangeable $\left.\mathrm{NH}\right) .2 \mathrm{~g}(80 \%)$; m.p. 268-270 ${ }^{\circ} \mathrm{C}$; MS (m/z): $304\left(\mathrm{M}^{+}\right)$; IR $\left(\mathrm{cm}^{-1}\right)$ : 3360-3100 (multiple bands, $2 \times \mathrm{xH}, \mathrm{NH}_{2}$ ) 1680, 1620 (br. bands, 2xCO); ${ }^{1} \mathrm{H}-\mathrm{NMR}(\delta): 3.75$ (3H, s, $\left.\mathrm{CH}_{3}\right), 4.36\left(2 \mathrm{H}, \mathrm{s}\right.$, exchangeable $\mathrm{NH}_{2}$ ) 7.79-8.17 (5H, a set of signals, $\mathrm{C}_{6} \mathrm{H}_{4}$ and pyrazole $\left.\mathrm{H}-3\right), 9.30$ (s, $1 \mathrm{H}$, exchangeable $\left.\mathrm{NH}\right), 10.79$ (1H, s, exchangeable NH). 2h (85\%); m.p. 244-245 ${ }^{\circ} \mathrm{C}$; MS (m/z): $293\left(\mathrm{M}^{+}\right)$; IR $\left(\mathrm{cm}^{-1}\right)$ : 3310, 3170 (br. $\mathrm{NH}$ and $\mathrm{NH}_{2}$ ) 1700, 1650 (2xCO); ${ }^{1} \mathrm{H}-\mathrm{NMR}(\delta): 3.72\left(3 \mathrm{H}, \mathrm{s}, \mathrm{CH}_{3}\right), 4.35(2 \mathrm{H}, \mathrm{s}$, exchangeable $\left.\mathrm{NH}_{2}\right) 7.52-7.89\left(5 \mathrm{H}\right.$, a set of signals, $\mathrm{C}_{6} \mathrm{H}_{4}$ and pyrazole $\left.\mathrm{H}-3\right), 9.20(\mathrm{~s}, 1 \mathrm{H}$, exchangeable $\mathrm{NH}), 10.49$ (1H, s, exchangeable $\mathrm{NH}$ ). 


\section{General procedure for 1-R-5-aminopyrazole-4-(N-benzoyl)carbohydrazides 5a,b,f}

A solution of 0.01 moles of a pyrazole-4-carbohydrazide $3 \mathbf{a}-\mathbf{f}$ in acetonitrile $(50 \mathrm{ml})$ was heated under reflux with 0.01 mole of the appropriate benzoyl chloride $\mathbf{6 a , b , f}$ for $7 \mathrm{~h}$. The solid which separated was collected then recrystallized from ethanol to give pure 5; yields 78-85\%. 5a (80\%) identical in all respect to the low-yield product obtained by reacting 4a with hydrazine hydrate. 5b (78\%); m.p. 263-265 ${ }^{\circ} \mathrm{C}$; MS (m/z): $355\left(\mathrm{M}^{+}\right)$; IR $\left(\mathrm{cm}^{-1}\right)$ : (Microanalysis Calc. for $\mathrm{C}_{17} \mathrm{H}_{14} \mathrm{~N}_{5} \mathrm{O}_{2} \mathrm{Cl} \cdot \mathrm{H}_{2} \mathrm{O}$ ): 3500-3100 (multiple bands, $\mathrm{NH}_{2}$ and $\mathrm{NH}-\mathrm{NH}$ ), 1660 (br., 2xCO); ${ }^{1} \mathrm{H}-$ NMR ( $\delta$ ): 6.45 (2H, br. s, exchangeable $\mathrm{NH}_{2}$ ), 7.41-7.98 (9H, a set of signals, $\mathrm{C}_{6} \mathrm{H}_{5}$ and $\left.\mathrm{C}_{6} \mathrm{H}_{4}\right)$, 8.07 (1H, s, pyrazole H-3), $9.99(1 \mathrm{H}$, s, exchangeable $\mathrm{NH}), 10.47(1 \mathrm{H}$, s, exchangeable $\mathrm{NH}) .5 \mathbf{f}$ (85\%); m.p. $275-276^{\circ} \mathrm{C}$ (ethanol); MS (m/z): $304\left(\mathrm{M}^{+}\right)$; IR ( $\mathrm{cm}^{-1}$ ) (Nujol): 3460-3100 (multiple bands, $\mathrm{NH}_{2}$ and NH-NH), 1685, 1620 (br., 2xCO); ${ }^{1} \mathrm{H}-\mathrm{NMR}(\delta)\left(\mathrm{DMSO}-\mathrm{d}_{6}\right): 3.54\left(3 \mathrm{H}, \mathrm{s}, \mathrm{CH}_{3}\right)$ $6.22\left(2 \mathrm{H}\right.$, br. s, exchangeable $\left.\mathrm{NH}_{2}\right), 7.42(1 \mathrm{H}, \mathrm{s}$, pyrazole $\mathrm{H}-3), 7.74-8.34\left(5 \mathrm{H}, \mathrm{dd}, \mathrm{C}_{6} \mathrm{H}_{5}\right), 9.83$ $(1 \mathrm{H}, \mathrm{s}$, exchangeable $\mathrm{NH}), 10.59$ (1H, s, exchangeable $\mathrm{NH})$.

1-Phenyl-5-benzamido-1H-pyrazolo[3,4-d]pyrimidine-4-one 7a. Method A. 1g (0.003 moles) of compound 5a and $10 \mathrm{ml}$ of triethyl orthoformate were heated at reflux for $4 \mathrm{~h}$. After cooling, the white solid product was collected and recrystallized from ethanol. 7a (75\%); m.p. 233-235 ${ }^{\circ} \mathrm{C}$; MS (m/z): $331\left(\mathrm{M}^{+}\right)$; IR ( $\mathrm{cm}^{-1}$ ): 3256 (br., NH), 1685 (br., CO); ${ }^{1} \mathrm{H}-\mathrm{NMR}(\delta): 7.43-8.66$ $\left(12 \mathrm{H}\right.$, a set of signals, $2 \mathrm{xC}_{6} \mathrm{H}_{5}$, pyrazole $\mathrm{H}-3$, quinazolinone $\left.\mathrm{H}-2\right), 11.93(1 \mathrm{H}$, s, exchangeable $\mathrm{NH})$.

Method B. Equimolar amounts (0.01 moles) of compound $\mathbf{8}^{\mathbf{8}}$ and the benzoyl chloride $\mathbf{6 a}$ in pyridine/dioxane (1:1) mixture were heated under reflux for $1 \mathrm{~h}$, then cooled and poured on cold diluted HCl. The solid which separated was collected, and recrystallized from ethanol to give pure 7a which was identical to that synthesized by method A (Rf, mixed m.p., MS); yields 53\%.

General procedure for ethyl 1-(R)-5-benzamido-1H-pyrazole-4-carboxylate 10b,d,f Equimolar amounts (0.033 moles) of ethyl 1-R-5-aminopyrazole-4-carboxylate $\mathbf{9 b , f}$ and the appropriate benzoyl chloride $\mathbf{6 b}, \mathbf{d}, \mathbf{f}$ in anhydrous acetonitrile $(150 \mathrm{ml})$ were heated at reflux for 5h. After the first hour, four portions of triethylamine, 2.2, 1.1, 0.6 and $0.6 \mathrm{ml}$ each, were added at intervals of $1 \mathrm{~h}$. The solution was evaporated in vacuum, the residue washed with water until it became solid, filtered off, and then recrystallized from ethanol to give compounds 10; yields 6580\%. 10b (78\%); m.p. 126-127 ${ }^{\circ} \mathrm{C}$; MS (m/z): $369\left(\mathrm{M}^{+}\right)$; IR ( $\left.\mathrm{cm}^{-1}\right)$ : 3400-3200 (NH), 1700, 1685 (2xCO); ${ }^{1} \mathrm{H}-\mathrm{NMR}(\delta): 1.14\left(3 \mathrm{H}, \mathrm{t}, \mathrm{CH}_{3}\right) 4.17\left(2 \mathrm{H}, \mathrm{q}, \mathrm{CH}_{2}\right)$ 7.24-8.20 (10H, multiplet, $\mathrm{C}_{6} \mathrm{H}_{5}$ $\mathrm{C}_{6} \mathrm{H}_{4}$ and pyrazole $\left.\mathrm{H}-3\right) 10.64$ (1H, s, exchangeable $\left.\mathrm{NH}\right) .10 \mathrm{10}$ (65\%); m.p. 148-150 ${ }^{\circ} \mathrm{C}$; MS (m/z): $307\left(\mathrm{M}^{+}\right)$; IR $\left(\mathrm{cm}^{-1}\right)$ : $3250(\mathrm{NH})$ 1710-1665 (2xCO); ${ }^{1} \mathrm{H}-\mathrm{NMR}(\delta): 1.15\left(3 \mathrm{H}, \mathrm{t}, \mathrm{CH}_{3}\right) 3.72$ $\left(3 \mathrm{H}, \mathrm{s}, \mathrm{CH}_{3}\right) 4.13\left(2 \mathrm{H}, \mathrm{q}, \mathrm{CH}_{2}\right)$ 7.65-8.06 (5H, multiplet, $\mathrm{C}_{6} \mathrm{H}_{4}$ and pyrazole $\left.\mathrm{H}-3\right) 10.56(1 \mathrm{H}, \mathrm{s}$, exchangeable NH). 10f (80\%); m.p. $140-142{ }^{\circ} \mathrm{C}$; MS (m/z): $318\left(\mathrm{M}^{+}\right)$; IR $\left(\mathrm{cm}^{-1}\right): 3330(\mathrm{NH})$, 1690-1670 (2xCO); ${ }^{1} \mathrm{H}-\mathrm{NMR}(\delta): 1.14\left(3 \mathrm{H}, \mathrm{t}, \mathrm{CH}_{3}\right) 3.32\left(3 \mathrm{H}, \mathrm{s}, \mathrm{CH}_{3}\right) 4.13\left(2 \mathrm{H}, \mathrm{q}, \mathrm{CH}_{2}\right) 7.98$ (1H, s, pyrazole H-3) 8.20-8.44 (4H, dd, $\left.\mathrm{C}_{6} \mathrm{H}_{4}\right) 10.82$ (1H, s, exchangeable $\left.\mathrm{NH}\right)$. 


\section{General procedure for 1-(R)-5-benzamido-1H-pyrazole-4-carboxylic acids 11b,d,f}

To a solution of ethyl 1-R-5-benzamido- $1 H$-pyrazole-4-carboxylate 10b,d,f (0.025 moles) in ethanol $(100 \mathrm{ml}) 120 \mathrm{ml}$ of an aqueous sodium hydroxide solution (4\%) was added. The mixture was heated at reflux for $15 \mathrm{~min}$. then allowed to stand at room temperature for $12 \mathrm{~h}$. The solution was cooled (ice bath) and dilute hydrochloric acid was added to give complete precipitation (pH 2). The separated solid was filtered off and recrystallized from ethanol to give 11b,d,f. Yields 80-85\%. 11b (80\%); m.p. 233-235 ${ }^{\circ} \mathrm{C}$; MS (m/z): $341\left(\mathrm{M}^{+}\right)$; IR ( $\left.\mathrm{cm}^{-1}\right)$ : 3300-2500 (OH, $\mathrm{NH}), 1690,1662$ (2xCO); ${ }^{1} \mathrm{H}-\mathrm{NMR}(\delta): 7.36-8.13\left(10 \mathrm{H}, \mathrm{C}_{6} \mathrm{H}_{5}, \mathrm{C}_{6} \mathrm{H}_{4}\right.$ and pyrazole H-3) 10.57 (1H, s, exchangeable $\mathrm{NH}) 12.57$ (1H, br. s, exchangeable $\mathrm{OH}) .11 \mathrm{~d}(85 \%)$; m.p. $205-207{ }^{\circ} \mathrm{C}$; MS (m/z): $279\left(\mathrm{M}^{+}\right)$; IR ( $\left.\mathrm{cm}^{-1}\right): 3590(\mathrm{OH}), 3320(\mathrm{NH}), 1700-1670(2 \mathrm{xCO}) ;{ }^{1} \mathrm{H}-\mathrm{NMR}(\delta): 3.69$ $\left(3 \mathrm{H}, \mathrm{s}, \mathrm{CH}_{3}\right)$ 7.65-8.06 (5H, multiplet, $\mathrm{C}_{6} \mathrm{H}_{4}$ and pyrazole $\left.\mathrm{H}-3\right) \mathrm{NH}$ and $\mathrm{OH}$ non-detectable signals. 11f (80\%); m.p. 227-230 ${ }^{\circ} \mathrm{C}$; MS (m/z): $290\left(\mathrm{M}^{+}\right)$; IR $\left(\mathrm{cm}^{-1}\right)$ : 3580-3320 (OH, NH), 1710-1680 (2xCO); ${ }^{1} \mathrm{H}-\mathrm{NMR}(\delta): 3.72\left(3 \mathrm{H}, \mathrm{s}, \mathrm{CH}_{3}\right) 7.86(1 \mathrm{H}, \mathrm{s}$, pyrazole $\mathrm{H}-3)$ 8.22-8.44 (4H, dd, $\left.\mathrm{C}_{6} \mathrm{H}_{4}\right), \mathrm{NH}$ and $\mathrm{OH}$ non-detectable signals.

General procedure for 1-(R)-6-(4-R - -phenyl)-pyrazolo[3,4-d]-1,3-oxazin-4-ones 4b,d,f Thionyl chloride $(10 \mathrm{ml})$ was added to a suspension of compound $\mathbf{1 1 b}, \mathbf{d}, \mathbf{f}$ (0.025 moles) in anhydrous benzene (45 ml). The mixture was heated at reflux for $4 \mathrm{~h}$, cooled, and the solid precipitate collected and recrystallized from ethanol; yields 68-75\%. 4b (70\%); m.p. $210{ }^{\circ} \mathrm{C}$; MS $(\mathrm{m} / \mathrm{z}): 323\left(\mathrm{M}^{+}\right)$; IR $\left(\mathrm{cm}^{-1}\right)$ : 1790-1780 (CO); ${ }^{1} \mathrm{H}-\mathrm{NMR}(\delta): 7.41-8.27\left(10 \mathrm{H}\right.$, complex, $\mathrm{C}_{6} \mathrm{H}_{5}$ $\mathrm{C}_{6} \mathrm{H}_{4}$ and pyrazole H-3). 4d (68\%); m.p. 203-205 ${ }^{\circ} \mathrm{C}$; MS (m/z): $261\left(\mathrm{M}^{+}\right)$; IR $\left(\mathrm{cm}^{-1}\right)$ : 1795-1785 (CO); ${ }^{1} \mathrm{H}-\mathrm{NMR}(\delta): 3.99\left(1 \mathrm{H}, \mathrm{s}, \mathrm{CH}_{3}\right), 7.67-8.27\left(5 \mathrm{H}\right.$, complex, $\mathrm{C}_{6} \mathrm{H}_{4}$ and pyrazole $\left.\mathrm{H}-3\right) .4 f$ (75\%); m.p. 215-220 ${ }^{\circ} \mathrm{C}$; MS (m/z): $272\left(\mathrm{M}^{+}\right)$; IR ( $\left.\mathrm{cm}^{-1}\right): 1780$ (CO); ${ }^{1} \mathrm{H}-\mathrm{NMR}(\delta): 8.29-8.40$ (5H, complex, $\mathrm{C}_{6} \mathrm{H}_{4}$ and pyrazole $\mathrm{H}-3$ ).

\section{Acknowledgments}

Financial support from MIUR is gratefully acknowledged.

\section{References}

1. $\quad$ Murad, S; Pinnel, S.R. PCT Int. Appl. 1992, WO 9217065 15.10.1002.

2. Mosshedy, M.; Ahmed, A.H.; El-Ghany, M.A. Alexandria Sci. Exch. 1986, 7, 33.

3. Wood, J.D.; Gorecki, D.K.; Dimmock, J. R.; Hawes, E.M. Can. J. Physiol. Pharmacol. 1975, 53, 47.

4. Klopman, G.; Fercu, D.; Jacob J., Chem. Phys. 1996, 204, 181.

5. Wamhoff, H.; Ertas, M.; Atta, S.M.S. Liebigs Ann. Chem. 1985, 1910. 
6. Daidone, G.; Plescia, F.; Maggio, B.; Raffa, D.; Cutuli, V.M.C.; Mangano, N.; Caruso, A. Arch. Pharm. Pharm. Med. Chem. 2001, 334, 153.

7. SDBSWeb: htpp://www.aist.go.jp/RIODB/SDBS/(access date).

8. Wamhoff, H.; Ertass, M.; Atta, Sana M.S. Liebigs Ann. Chem. 1985, 1910.

9. Daidone, G.; Bajardi, M.L.; Roccaro, A.; Raffa, D.; Caruso, A.; Cutuli, V.; Di Pietro, E. Il Farmaco 1991, 46, 945.

10. Still, W.C.; Kahn, M.; Mitra, A. J. Org. Chem. 1978, 43, 2923. 